\title{
Ablation laser femtoseconde du carbone : étude du panache plasma et dépôt de couches minces
}

\author{
F. Garrelie, C. Jonin, A. Catherinot ${ }^{1}$, C. Champeaux ${ }^{1}$, E. Baubeau, E. Audouard, \\ S. Mottin et P. Laporte
}

\author{
Laboratoire Traitement du Signal et Instrumentation, Université Jean Monnet, \\ UMR 5516 du CNRS, 23 rue du Docteur Paul Michelon, 42023 Saint-Etienne cedex 2, France \\ ${ }^{1}$ Laboratoire Science des Procédés Céramiques et de Traitements de Surface, \\ UMR 6638 du CNRS, 123 avenue A. Thomas, 87060 Limoges cedex, France
}

\begin{abstract}
Résumé: Le transport du panache plasma créé par ablation laser femtoseconde d'une cible de carbone est étudié par imagerie ultra-rapide. L'énergie cinétique des particules émettrices ainsi détectées est voisine de $2 \mathrm{keV}$ pour les plus rapides d'entre elles (détectables pour des délais inférieurs à $100 \mathrm{~ns}$ après l'impulsion laser), et de $100 \mathrm{eV}$ pour la composante la plus lumineuse du panache plasma (détectable jusqu'à $1 \mu$ s après l'impulsion laser). Des couches minces de DLC ont également été élaborées par ablation laser femtoseconde. Le film obtenu à une fluence de $0.5 \mathrm{~J} / \mathrm{cm}^{2}$ présente par exemple un taux d'hybridation $\mathrm{sp} 3$ du carbone voisin de $55 \%$.
\end{abstract}

\section{INTRODUCTION}

Le procédé de dépôt de couches minces par ablation laser (PLD) suscite un vif intérêt depuis de nombreuses années tant pour des applications microélectroniques qu'optiques ou mécaniques. En particulier, de nombreuses équipes ont entrepris de déposer des couches minces de carbone amorphe (DLC ou ta-C) $[1,2]$ par ablation laser, le plus souvent en utilisant des lasers excimères fonctionnant en régime nanoseconde. Une forte corrélation entre la structure des couches obtenues et l'énergie cinétique des particules du plasma a été observée [3]. L'utilisation d'un laser de durée d'impulsion femtoseconde semble conduire à des énergies cinétiques des particules très supérieures à celles obtenues lors de l'ablation par des lasers de durée d'impulsion nanoseconde [4]. Jusqu'à présent, les caractérisations du panache plasma créé par ablation laser femtoseconde d'une cible de carbone ont été obtenues uniquement par des techniques de mesure de temps de vol des ions (TOF) $[4,5]$.

Dans cet article, nous présentons une étude expérimentale de l'ablation par un laser femtoseconde d'une cible de carbone. Des photographies résolues en temps et spatialement du panache plasma sont présentées, afin d'accéder à la répartition spatiale et à l'énergie cinétique des particules. Des couches minces de carbone amorphe ont également été élaborées dans ces conditions.

\section{ETUDE DU PANACHE PLASMA}

Un laser saphir : Ti (Alpha 1000, BMI/TCL) de longueur d'onde $800 \mathrm{~nm}$, délivrant une énergie par impulsion de $0.4 \mathrm{~mJ}$ avec une durée d'impulsion de $150 \mathrm{fs}$ à un taux de répétition de $1 \mathrm{kHz}$, est focalisé sur une cible de graphite placée dans une enceinte ultra-vide. La fluence laser correspondante est de 2 $\mathrm{J} / \mathrm{cm}^{2}$. Les mesures ont été effectuées sous une pression résiduelle d'air de $5.10^{-3} \mathrm{~Pa}$. Le comportement spatio-temporel du panache plasma créé par ablation laser femtoseconde de la cible de carbone est examiné en utilisant une caméra CCD intensifiée à ouverture rapide (Princeton Instruments). L'imagerie résolue en temps et spatialement permet d'accéder à la répartition spatiale mais également à l'énergie cinétique des espèces émettrices du plasma (domaine spectral : $350-800 \mathrm{~nm}$ ).

La figure 1 représente des images du panache plasma à différents délais après le début de l'impulsion laser. Chaque image correspond à l'accumulation des signaux sur 10 impulsions laser, la taille de chaque image est de $2 \times 2 \mathrm{~cm}^{2}$.

Plusieurs observations peuvent être effectuées. Tout d'abord, d'un point de vue qualitatif, nous observons plusieurs composantes dans le panache plasma. Pour des délais inférieurs à 100 ns après l'impulsion laser, les particules émettrices sont éjectées de la cible avec une énergie cinétique très élevée, puis une seconde composante peut être détectée jusqu'à un délai d'environ $1 \mu$ s après l'impulsion laser. D'un point de vue 
quantitatif, en reportant l'évolution temporelle de la hauteur atteinte par le front du panache plasma, la valeur de l'énergie cinétique des particules émettrices ainsi observées est voisine, respectivement, de $2 \mathrm{keV}$ pour les particules les plus rapides, et de $100 \mathrm{eV}$ pour la seconde composante.
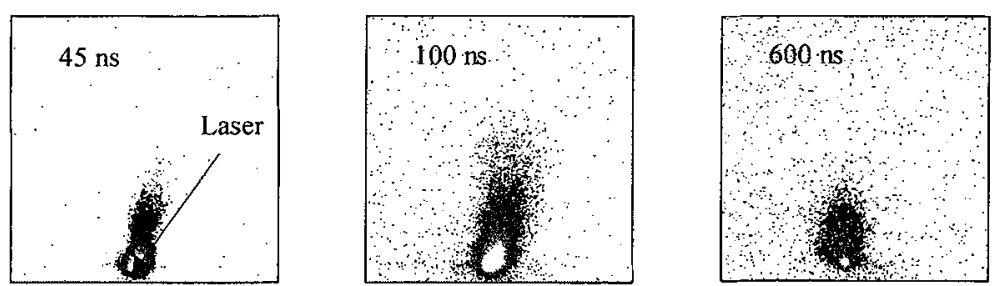

Figure 1: Images du panache plasma créé par ablation laser femtoseconde d'une cible de carbone, à différents délais après le début de l'impulsion laser (temps d'intégration de chaque image respectivement de $40 \mathrm{~ns}, 50 \mathrm{~ns}$ et $100 \mathrm{~ns}$ pour les délais de 45 et $100 \mathrm{~ns}$ et $600 \mathrm{~ns}$ ).

\section{DEPOT DE COUCHES MINCES DE DLC}

Des couches minces de DLC ont également été déposées, sous vide sur substrat de silicium monocristallin, par ablation laser femtoseconde d'une cible de graphite et ce pour différentes valeurs de fluence laser. La densité surfacique de particules de taille micronique ("escarbilles") sur la couche mince est très inférieure à celle obtenue lors du dépôt par ablation par laser excimères nanoseconde. Par ailleurs, à titre d'exemple, un film obtenu à une fluence laser de $0.5 \mathrm{~J} / \mathrm{cm}^{2}$, présente un taux d'hybridation $\mathrm{sp}^{3}$, déterminé par analyse XPS, voisin de $55 \%$.

\section{CONCLUSION}

Nous avons présenté une étude expérimentale de l'ablation d'une cible de graphite par un laser de durée d'impulsion femtoseconde. Des photographies du panache plasma de matière éjectée ont été présentées, permettant ainsi de déterminer l'énergie cinétique des particules. La composante la plus rapide des particules émettrices est éjectée avec une énergie cinétique de l'ordre de $2 \mathrm{keV}$, la composante principale possédant quant à elle une énergie cinétique de l'ordre de $100 \mathrm{eV}$. Des couches présentant un taux d'hybridation $\mathrm{sp}^{3}$ du carbone voisin de $55 \%$ ont également été présentées. Du fait de ces résultats encourageants, une étude plus systématique des propriétés des couches minces de DLC obtenues par ablation laser femtoseconde en fonction de l'énergie des particules éjectées de la cible a été entreprise.

\section{Remerciements}

Les auteurs remercient K. Wolski (Ecole des Mines - Saint Etienne) pour l'analyse XPS des couches.

\section{Références}

1. Pulsed Laser Deposition of Thin Films, edited by D.B. Chrisey, C.K. Hubler (Naval Research Laboratory, Washington, DC, 1994).

2. C. Germain, C. Girault, J. Aubreton, A. Catherinot, S. Bec and A. Tonck, Diamond Relat. Mater. 4 (1995), 309.

3. V.I. Merkulov, D.H. Lowndes, G.E. Gellison, Jr, A.A. Puretzky and D.B. Geohegan, Appl. Phys. Lett. 73 (1998), 2591.

4. F. Qian, V. Craciun, R.K. Singh, S.D. Dutta and P.P. Pronko, J. Appl. Phys. 86 (1999), 2281.

5. P.S. Banks, L. Dinh, B.C. Stuart, M.D. Feit, A.M. Komashko, A. M. Rubenchik, M.D. Perry and W. McLean, Appl. Phys. A 69 (1999), S347. 